\title{
Appraising the Clinical Efficacy of Herbal Topical Medications in the Treatment of Recurrent Aphthous Stomatitis - A Review
}

\author{
Kavitha Muthu1, ${ }^{1,}$, Deepshika Saravanan ${ }^{2}$ Saravanan Rethinam ${ }^{3}$, Sathyasree Madeswaran ${ }^{4}$, Nanditha Suresh ${ }^{5}$ \\ 'Department of Oral Pathology, RVS Dental College and Hospital, Coimbatore, Tamil Nadu, INDIA. \\ 2Department of Periodontology, RVS Dental College and Hospital, Coimbatore, Tamil Nadu, INDIA. \\ ${ }^{3}$ Department of Oral and Maxillo Facial Surgery, RVS Dental College and Hospital, Coimbatore, Tamil Nadu, INDIA. \\ ${ }^{4}$ Department of Prosthodontics and Crown and Bridge, RVS Dental College and Hospital, Coimbatore, Tamil Nadu, INDIA. \\ Department of Periodontology, Asan Dental College and Hospital, Chennai, Tamil Nadu, INDIA.
}

\begin{abstract}
Oral ulcers are common complaints to the dental clinic. Among these, Recurrent Aphthous Stomatitis/Ulcer (RAS) is frequently reported lesions of which recurrent minor aphthous ulcers are most prevalent. Though these mucosal lesions are self-limiting, painful ulcerative and inflamed mucosa could affect the patient's daily routine such as food intake, speaking and swallowing. Since precise etiology is unknown, various treatment options are tailor made to each individual patient's condition. Topical steroids are the widely prescribed medication as local therapy. In spite of a dearth in evidence based data on the effectiveness of plant based topical therapies for oral aphthous ulcers, these are used as primary medications which aids in natural cure of such lesions. These alternative medications are also useful to patients who refuse to take allopathic medicines, having history of drug allergies, history of multiple drug therapy, when allopathic medication is not recommended and in refractory cases. Hence the aim of this paper is to review the clinical efficacy of potential herbal medications for the topical management of recurrent aphthous ulcers. An electronic data search
\end{abstract}

was performed using different search engines and the relevant articles were reviewed. All the placebo controlled studies have shown clinically significant results in the treated group in various outcome measures without noticeable side effects. Generally, topical herbal treatment were effective on RAS as palliative therapy, however more scientific evidence is needed for clinical recommendation in repeated cases.

Key words: Aphthous stomatitis /ulcer, Alternative medications, Topical herbs, Topical treatment, Recurrent oral ulcer,Herbal medications.

\section{Correspondence}

Dr. Kavitha Muthu BDS, MDS.

Reader, Department of Oral Pathology, RVS Dental College and Hospital,

Coimbatore-641402, Tamil Nadu, INDIA.

Email id: kavidentist@yahoo.co.in

DOI: 10.5530/jyp.2021.13.87

\section{INTRODUCTION}

In recent times, consciousness towards the utilization of bio friendly and eco-friendly plant based products for the prevention and remedy of various human illnesses has paid considerable attention. The faith towards the herbal medicines has been growing worldwide. The phytochemical found in plants have protective and disease preventive properties which are non-nutritive in nature. ${ }^{1}$

Recurrent Aphthous Stomatitis (RAS) is considered as a mucosal disease which occurs locally and not a result of systemic disease. Since there is any definitive recommended treatment protocol for aphthous ulcer, patients are advised to attempt various medications, particularly in recurrent cases of aphthous ulcer. Many herbs which have potential for symptomatic treatment of aphthous are lacking scientific evidence for clinical use. Though many potential herbs were identified through ethno biological survey, the therapeutic isolation and clinical application of the herbal components are still lacking. Few herbs were tried on animal models and lab studies on human cells.

The recurrent ulcers appear periodically and heal completely between attacks. These ulcers have comparatively few common reasons. The common recurrent oral ulcers include recurrent aphthous ulcer (RAU), erythema multiforme, occasional traumatic ulcers and ulcers associated with gastro intestinal diseases. ${ }^{2} \mathrm{RAU}$ otherwise referred to as the common canker sore is a painful ulcerative lesion of an uncertain etiology. These recurring ulcers are $\mathrm{T}$ cell mediated solitary or multiple lesions, frequently seen in childhood and adolescent females.
Nutritional deficiencies, inflammatory bowel diseases, celiac disease, Behcet's syndrome, HIV are few conditions associated with aphthous stomatitis. The clinical forms include recurrent aphthous minor, recurrent aphthous major and recurrent herpetiform ulcerations. The minor ulcers commonly involve non keratinized mucosa with ulcers ranging from 3-10 $\mathrm{mm}$ diameter and resolve within 1-2 weeks. The major ulcers usually appear on the detached buccal and labial mucosa with more than 1 centimeter in diameter and heal in six weeks' time. However, it has been suggested that these ulcers respond poorly to any kind of therapies. In many patients, resolution of the systemic disorder gives rise to a reduced incidence and severity of the mucosal ulcerations. ${ }^{2,3}$ The mucosal destruction in RAS patients appears to represent a T cellmediated immunologic reaction with production of tumor necrosis factor-alpha. However, the initiating causes are variable. It has been theorized that aphthous ulcerations develop from an immunologic response to an oral antigen. This reaction may arise due to the presence of a highly antigenic reagent, a decrease in the mucosal barrier that previously masked the antigen, or immune dysregulation resulting in an abnormal response to a normally present antigen. The genetic factors, oral microbial flora and immunological factors plays major role in the etiology of RAS. Though there is multifactorial etiology, trauma, stress, allergy and smoking are considered as major predisposing factors. ${ }^{4-6}$

The major objective of the treatment is to reduce pain, amount and size of lesion, decrease the super infection, fastening the healing time and to

This is an open access article distributed under the terms of the Creative Commons Attribution-NonCommercial-ShareAlike 4.0 License, which allows others to remix, tweak, and build upon the work non-commercially, as long as the author is credited and the new creations are licensed under the identical terms. 
increase disease-free periods. The various treatment options comprise of topical therapies, intralesional injections, laser therapy, systemic therapy and botulinum toxins. The agents used for topical treatment should have easy application, tolerable, acceptable taste; best muco-adhesive properties with less side effects. ${ }^{6,7}$ These are mostly aimed for treating the current ulcers. Topical steroids are commonly prescribed medication for local therapy. Herbal topical agents have the advantages such as cheaper in cost, less side effects, easy availability with better patient acceptance. Some of these drugs also have the advantage of systemic administration along with local applications in needed circumstances. Table 1 describes the topical herbal medicines used in clinical trial for the treatment of Recurrent Aphthous Stomatitis. ${ }^{8-29}$

The significance of this review is to identify the potential herbal medicines utilized for topical management of RAS, to appraise the clinical effectiveness of topical herbal medicine in treating RAS and to identify the properties and mechanism of action of herbal topical medicine in treating RAS.

\section{MATERIALS AND METHODS}

Electronic databases such as MEDLINE-PubMed, GOOGLE SCHOLAR AND SCIENCE DIRECT were explored for the literature search on topical applications of herbal medicine in treating Recurrent Aphthous Stomatitis. MeSH terms such as aphthous ulcer, herbal treatment, randomized clinical trials, topical applications, alternative medications were used. Publication types i.e. reviews and original research articles with Randomized clinical Trial were sourced. Age, sex, geographical distribution restrictions were not set. Case reports and articles published in other languages were omitted from the pool. Supplementary data was collected from reference list of articles and other relevant articles. By using filters, articles were sourced from years 2008 -2018.

Two reviewers were assigned to choose and draft the articles with a set of suitability principles and for the valuation of articles that fit the criteria.

Table 1: Topical herbal medicines used in clinical trial for the treatment of Recurrent Aphthous Stomatitis. ${ }^{8-29}$

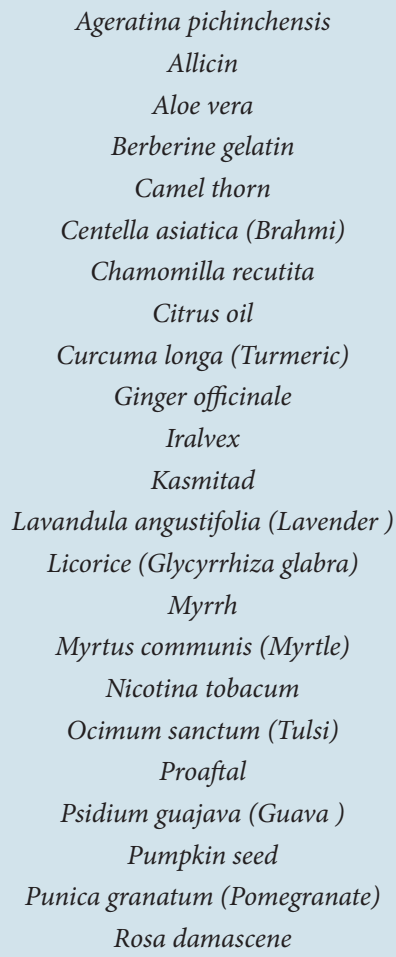

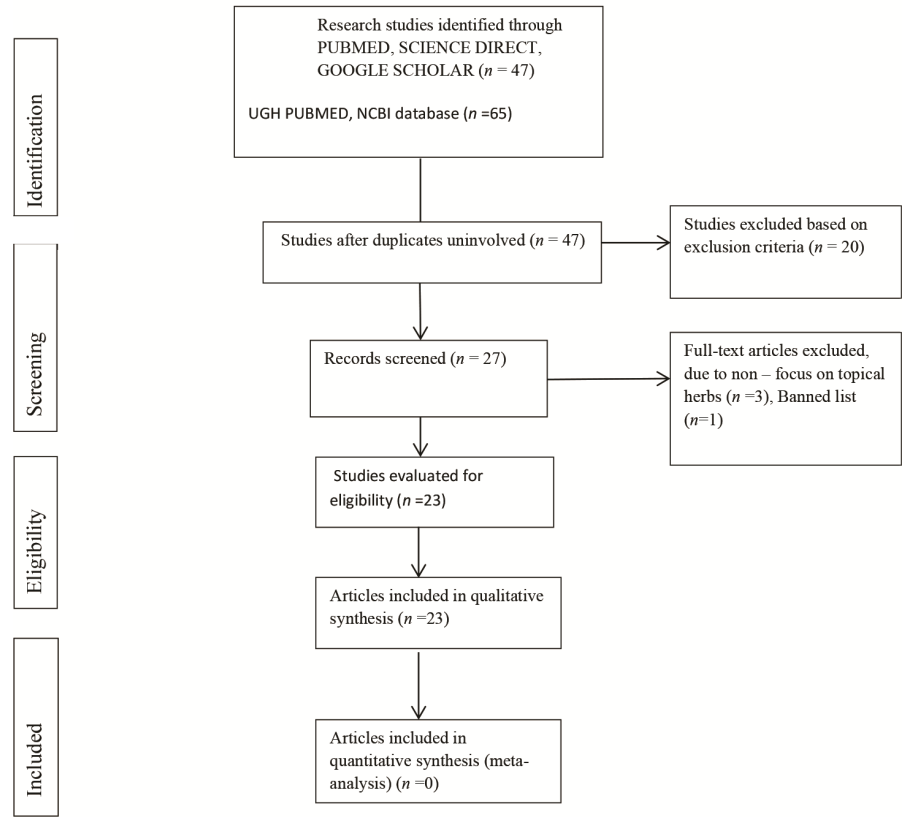

Figure 1: The flow diagram depicts the method of selection of articles for the review.

The reviewers were un-blinded and some difference among them was organized through acceptance between all five authors. After removing the duplication of articles, the review was done with the abstracts and full text of accessible ones. As abstracts only articles and different study designs of Randomized Clinical Trial (RCT) were included in this study while appraising the positive outcomes, some amount of recording bias could be anticipated. Figure 1 Flowchart.

\section{RESULTS}

The following data were collected from each included study

(1) Name of the herb and preparation method (2) Author (3) study cohort

(4) Number, amount and duration of the topical application

(5) Outcome measures such as effects on pain, healing time, and size of the ulcers, number of ulcers, erythema, exudation, recurrence rate and side effects.

(6) Relevant statistical analysis of study results.

The reviewers depicted their suggestions against each study based on the above mentioned data. Only 23articles that fulfilled the inclusion criteria from a total of 47 were included. In descending order all the data are presented consecutively from the articles beginning from year 2018 until 2008 with summaries in Table $2 .^{2-26}$

Most of the studies were from Asian region in which majority of the studies are from Iran (9) and others include North America, Africa and Europe region. Among the 23 studies, four studies were active controlled, 14 were placebo controlled; another five studies were self-controlled studies. Regarding the form of application, mouthwash/mouth rinse was the commonly used topical preparation followed by gel preparation. Mucoadhesive patch/membrane is the recently explored form of topical herbs. Majority of the studies utilized preparations with single herb $(69.6 \%)$ and remaining studies $(30.4 \%)$ used combined preparations. None of the studies showed any noticeable side effect. Maximum of the studies focused on effect of herbal preparations over aphthous stomatitis minor. All the placebo controlled studies have shown clinically significant 


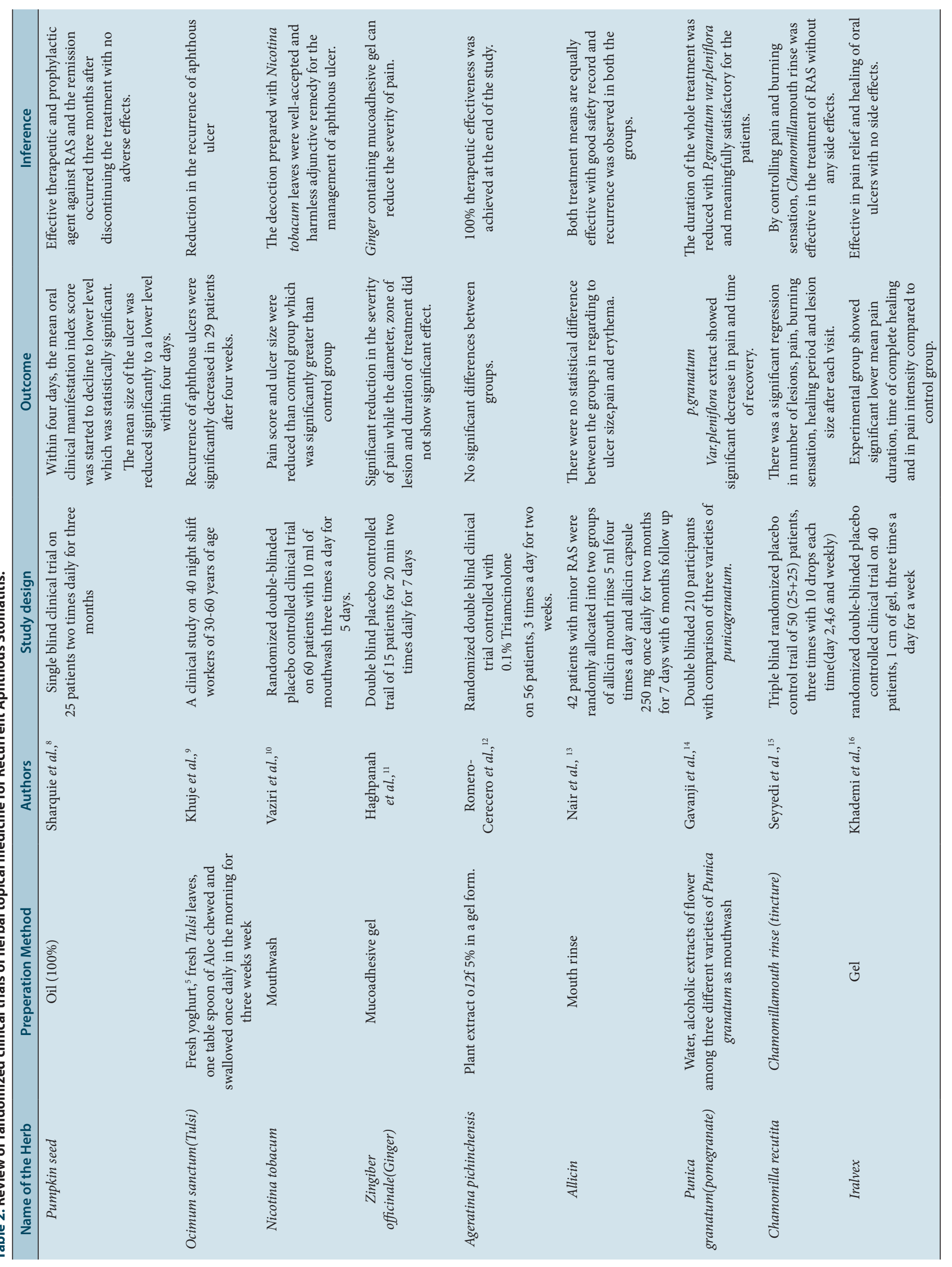




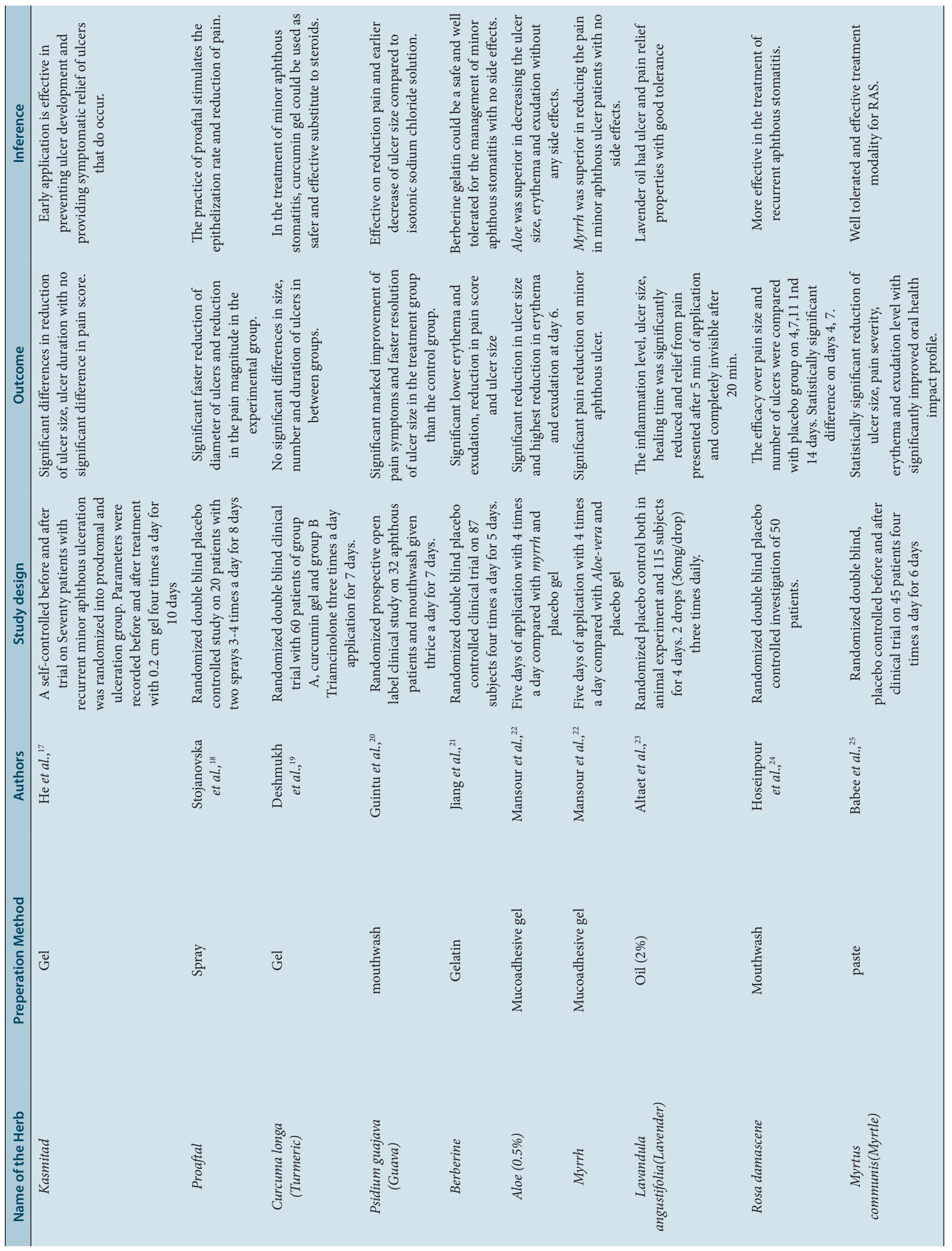




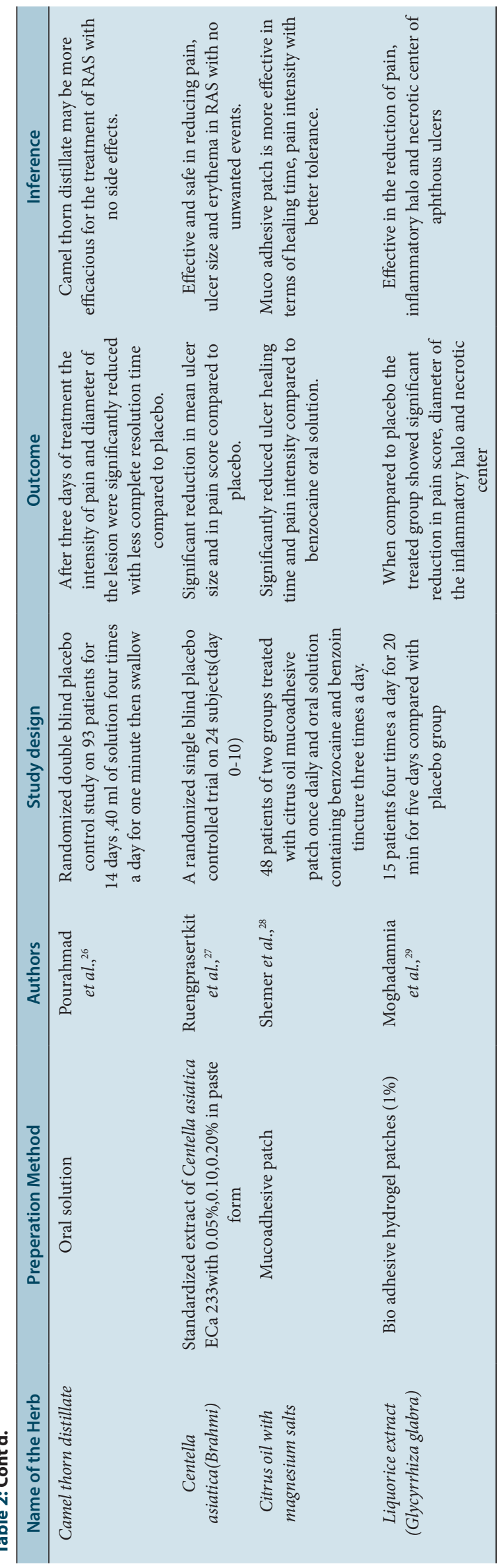

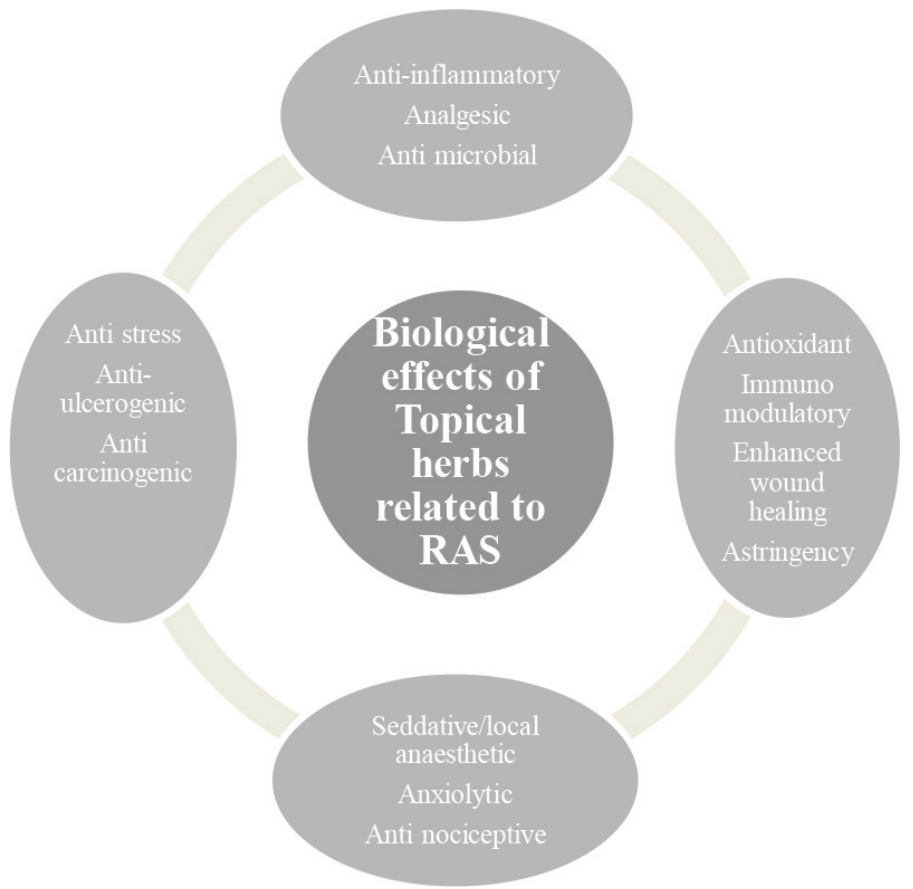

Figure 2: Biological effects of topical herbs related to RAS.

results in the treated group in various outcome measures. Two of the active controlled studies showed no significant results among the groups and another two studies have shown statistically significant results. The self-controlled trials also have presented clinically significant results on various tested parameters in aphthous ulcer patients within the study limits. All the studies primarily focused on symptomatic treatment of RAS between test and control groups, and topical herbal treatment was effective on reducing the symptoms. Whereas, two studies also focused topical herbs (pumpkin seed oil, Kasmitad gel) as a prophylactic/ preventive therapy and proved to be effective in preventing the ulcer development during the study period. Two studies (Allicin, Ocimum sanctum) included both topical and systemic intake as study component and the recurrence of ulcer was also included as an outcome measure. A comparative study stated recurrence of aphthous ulcer occurred in both the forms of (mouth rinse and capsule) treatment. The study on Ocimum sanctum showed a statistically significant result in reduction in the recurrence of aphthous ulcer. This might be due to the combined herbal therapy as well as the systemic effect of the herbs.

Figure 2 represents the general biological effects of herbal drugs related to topical treatment of RAS.

\section{DISCUSSION}

\section{Pumpkin seed oil (Cucurbitapepo L)}

Vitamins, minerals and anti-oxidants are present in huge amounts in pumpkin seed oils. The other active ingredients comprise fatty acids in particular high content of polyunsaturated fatty acids, tocopherol, carotenoids, phytosterols and few amino acids. It has antioxidant, antiinflammatory, antimicrobial and anticarcinogenic effect. A recent animal study in rats revealed better cutaneous wound healing in pumpkin oil extract treated group compared to untreated groups. The pumpkin oil treated group in healed biopsies showed a complete re-epithelialization with re-emergence of skin appendages and also well-organized collagen fibers with the absence of inflammatory cells. ${ }^{30}$ 


\section{Tulsi (Ocimum sanctum)}

The chief active ingredients in Tulsi comprises eugenol and ursolic acid, $\beta$-caryophyllene, flavonoids with little bioactive components include methyl chevicol, linalool and 1,8-cineole. The nutritional content of its seeds include carbohydrates, fibre, fats, important vitamins and minerals. Many scientific studies have proved that Ocimum sanctum has anti-bacterial, antifungal and antiviral properties. It also has got immunomodulatory activity and induces cytokine secretion. In addition, anti-toxic, antitussive, hepatoprotective, hypoglycemic, hypolipidemic, chemo preventive, anti-estrogenic properties have also been proved with wide margin of safety. The rapid elimination, low bio availability and perceived toxicity make this herb a potential therapeutic agent. ${ }^{31}$

It has been proved that Ocimum sanctum reduces the gastric ulcer is dosedependent manner. The anti-ulcer effect is mainly due to enhancement of antioxidant potential of gastric mucosa. Fixed oil and linolenic acid existing in tulsi are known to block cyclooxygenase and lipoxygenase pathways of arachidonic acid metabolism. ${ }^{9}$ Ocimum sanctum at a dose of $100 \mathrm{mg} / \mathrm{kg}$ was found to be effective antiulcer agent. This is due to its cytoprotective effect. The wound healing nature of Ocimum sanctum is mainly due to its anti-inflammatory, antimicrobial, anti-oxidant and immunomodulatory properties.

Tulsi is also known for its anti-stress activity by being able to control the balance of various metabolic processes of the body and prevent stress. The immuno-stimulant capacity of the herb helps in the adaptogenic activity. The aqueous, alcoholic, chloroform and essential oil extracts of leaves were equally effective against pathogenic gram positive and gram negative bacteria which also includes staphylococcal aureus. ${ }^{32}$ Several animal studies concluded that the oral doses of tulsi aqueous extract enhances the RBC, WBC, hemoglobin and antibodies without affecting the bio-chemical parameters. ${ }^{33}$

\section{Nicotina tobacum}

The leaves of the Nicotina tobacum have got emetic, narcotic, sedative, antispasmodic activities and have been utilized for the skin disorders and rheumatic swelling. Isoflavones, phenolic acids, sesquiterpenes, diterpenoids and alkaloids are the important biological compounds found in Nicotina tobacum. ${ }^{34}$ Literature report supports that smoking has protective activity in the occurrence of ulcer. The increased keratinization of oral mucosa is the consequence of the combustible products of smoking that could resist the formation of aphthous ulcers, decrease the microbial infiltration and trauma in smokers when compared to nonsmokers. Nicotine and its metabolites lead to reduction in inflammatory condition by exhibiting immunosuppressive therapy through various mechanisms. In addition, the inhibitory effect of the leaf extracts on diverse bacterial species effects in improvement of disease process. ${ }^{10}$

\section{Ginger (Zingiber officinale)}

Ginger has been used as a common herb in traditional medicine. Ginger a rhizome of zingiber officinale, is well known for its anti-inflammatory and anti-emetic effect. The sedative and pain regulator activity results from substance P release. Gingerols, shogaols, zingerone, paradol, gingerenone, galanal, gingerdiols and gingerdiones are the various bioactive ingredients reported from rhizomes. The gingerols and shogaols are known to be mutagenic. ${ }^{35}$ A clinical study suggested that the dried ginger rhizome membrane is effective in pain relief, reducing the healing time of aphthous ulcers. It also reduces the salivary EGF levels and preventive influence on TNF- $\alpha$ release. ${ }^{36}$

\section{Ageratina pichinchensis}

Ageratina pichinchensis extract proves anti-inflammatory, antinociceptive and wound healing properties in various experimental and clinical studies. It contains phytochemicals like encecalin, 7-0-( $\beta-D-$ glucopyranosyl)-gossypetin and 7-0-( $\beta$-D-glucopyranosyl)-galactin. Among this encecalin comprises a majority compound while galactin is identified as a wound healing promoting compound. This component was capable of constantly encourage cellular proliferation of normal human skin cells (HFS-30). ${ }^{12}$

\section{Allicin mouth rinse}

The chief constituent of garlic extracts is allicin. It shows antiinflammatory, anti-oxidation, anti-microbial, and immunomodulation characteristics. In animal models, topical allicin promoted healing process of oral ulcer by promoting re-epithelialization. The bioactive components of allicin reach the cell via phospholipid membrane and the ulceration provides easy diffusion of allicin into the wound. It is known to increase the blood supply to the area by promoting the elastic property of blood vessel and capillary perfusion. The antimicrobial property is responsible for the decreased bacterial colonization that prevents wound infection. In vascular endothelial cells, glutathione production is up-regulated and pro-inflammatory cytokines level is down regulated by allicin thereby decreasing excessive inflammatory process associated with RAS and speeds the healing. ${ }^{37}$

\section{Pomegranate (Punica granatum)}

Pomegranate extracts are having enormous medicinal value. Pomegranate is rich in tannins and polyphenols such as ellagitannins, punicalagins, punicallin and gallotannin. These polyphenols has antibacterial, antioxidant, anti-inflammatory, antiproliferative and DNA repair activities. The punicalagin exerts anti-inflammatory action by inhibiting the nuclear factor kappa B. (NF-kB) and by prevention of ERK-1 or ERK2 (mitogen-activated protein kinase cascades) activation. Pomegranate extracts also inhibits nitric oxide (NO) production by RAW 264.7 macrophage cells. The poly phenols from pomegranate extract have an inhibitory effect on IL-6 and IL-8. The punicalagin shows antioxidant property by increasing Nrf2-mediated HO-1 expression and also induces the P13K/Akt -mediated HO-1 expression. Punicalagin enhances the SOD1 mRNA expression and thereby inhibits reactive oxygen species generation and $\mathrm{NO}$ over production by macrophages. The tannins and polyphenols also induce fibroblast migration and proliferation, collagen formation and angiogenesis in wound healing. During the healing process, the pomegranate-treated wounds displayed better epithelialization and contractions of incised wounds with improved hydroxyproline content, dry weight and breaking strength of granulated tissues. ${ }^{38}$

\section{Chamomilla recutita}

As an alternative herbal medicine the Chamomilla has anti-inflammatory, antispasm, antibacterial, antifungal and analgesic properties and have been used in many inflammatory lesions. The essences (azolene, camazoline) and flavonoids are the two main ingredients of chamomilla. Camazolene has dose dependent anti-inflammatory and antispasm effects by inhibiting synthesis of leukotriene B4 [LTB4] synthesis and arachidonic acid peroxidation in neutrophils. Azolene possesses antiinflammatory and analgesic properties. Flavonoids are also recognized to have anti-inflammatory properties. Chamomile also comprises bisaboloids, terpenoids, matricine, chamazulene and cumarins. The antioxidant potential of chamomile is responsible for the ulcer healing. The anti-inflammatory effects of flavonoid is due to the inhibition of STAT-1 and NF- $\kappa \mathrm{B}$ activations and also the preventive effect on iNOS expression and $\mathrm{NO}$ formation in activated macrophages. ${ }^{39}$

\section{Iralvex}

Iralvex gel consists of $170 \mathrm{mg}$ dried rhubarb extract and $10 \mathrm{mg}$ of salicylic acid. The rhubarb extract comprises of anthraquinones, 
anthrone, dianthrones, heterodianthrones, tannins, starch and calcium oxalate. Antraquinoneglycosides, tannin and salicylic acid in the gel react with the proteins of the mucous membrane epithelial cells leading to a tougher adhesion of mucosa which results in a decline in the penetrability of the cells. Gingival mucosal outer layer is thus protected against microbes and toxins by the astringency process. These chemicals also have antimicrobial effects that lead to better pain relief and earlier wound healing. ${ }^{16}$

\section{Kasmitad gel}

Kasmitad gel is a compound preparation by STADA pharmaceutical company and approved in 1978 for production in Germany. It has been used for topical treatment of recurrent oral ulcers which consists of $200 \mathrm{mg}$ chamomile flower tincture (1:5.5), $20 \mathrm{mg}$ lidocaine, $1 \mathrm{mg}$ thymol and $1 \mathrm{mg}$ of benzalkonium chloride as a preservative. Chamomile tincture has anti-inflammatory, antimicrobial and antioxidant effect. When combined with lidocaine it shows analgesic effect. Studies have shown that the gel provides immediate pain relief. ${ }^{17}$

\section{Proaftal}

Proaftal is made up of propolis and essential oils which comprises antiinflammatory ingredients of plant origin. This contains $25 \%$ dry matter propolis, etheric oils from sage mint, anise, menthol mixed in $100 \mathrm{ml}$ of diluted alcohol. Propolis is well recognized for its antimicrobial effects against certain bacteria, fungi and viruses etc. Flavonoids, aromatic acids, esters, aldehydes, ketones, fatty acids, steroids, terpenes, amino acids, polysaccharides, hydroxybenzene, alcohol etc. are included in the chemical composition of propolis. ${ }^{40}$ The flavonoids are found to strengthen the capillary walls and reinforce the association of serum proteins with histamine. Flavonoids also have anti-oxidant effect. Being a local anesthetic, propolis also regenerates and heals tissue, increases the value of properdin in serum which accounts for its immunomodulatory effects and stimulates alternative pathways for the complement system. ${ }^{18}$

\section{Turmeric (curcuma longa)}

The main ingredient of turmeric is curcumin which is proved to be nontoxic in nature. It has showed diversified effect $s$ in various oral diseases. It has got antioxidant, anti-inflammatory, anti-microbial, immunomodulatory, chemopreventive properties. The anti-inflammatory properties are due to the inhibiting activity of $\mathrm{NF}-(\kappa) \mathrm{B}$ transcription factor, reducing the production of TNF- $\alpha$ and IL- 1 cytokines. The properties like low bio availability, rapid elimination and the less perceived toxicity are the therapeutic benefits of curcumin. Regarding wound healing properties, it has been inferred that it increases the micro circulation, increases angiogenesis, improves the granulation tissue formation and accelerates re-epithelialization. ${ }^{41}$ It has been suggested that curcumin gel is a well-tolerated and effective treatment modality for aphthous. Curcumin holds limitations like low solubility, rapid metabolism and hence low bio availability. ${ }^{19}$

\section{Guava leaves (Psidium guajava)}

P. guajava possesses antimicrobial, anti-inflammatory, antitumor, antiallergic, antihyperglycemic and antimutagenic characteristics. Wounds, cough and dental diseases has been treated by P. guajava. It contains huge phytochemicals and antioxidants comprising polysaccharides, essential oils, minerals, vitamins, enzymes, alkaloids, steroids, flavonoids, tannins, triterpenoid acid, glycosides, saponins and other chemical compounds. Flavonoids have anti-inflammatory and analgesic actions. ${ }^{42}$ They inhibit the biosynthesis of prostaglandins and inhibit protein kinase enzyme thereby producing anti-inflammatory effect. ${ }^{20}$

\section{Berberine gelatin}

Hydrastis Canadensis, Coptidis rhizome and berberis vulgaris are the medicinal herbal source of berberine. It is an isoquinoline alkaloid isolated from these plants. It possesses anti-inflammatory, antimicrobial, antitumour and anti-pyretic properties. Studies showed that via activated protein kinase activation in macrophages, berberine could inhibit pro inflammatory responses. It also downregulates the expression of pro-inflammatory genes (TNF $-\alpha$, interleukin- $1 \beta$, IL-6 etc.) and upregulates IL-10 which has major negative regulatory feedback on Th-1 cytokine production. Berberine gelatin contains $50 \mathrm{mg}$ berberine, hydroxypropyl methylcellulose, polyethylene glycol 400, glycerine and flavoring additives. Berberine is known for its antimicrobial activity against Staphylococcus, Streptococcus, Candida and Salmonella. In addition, berberine is an immunosuppressive agent with safe drug profile. ${ }^{21}$

\section{Aloe vera}

Aloe vera contains many essential amino acids which are needed by our body and these amino acids aids in smooth functioning of our complex enzyme system. It is well known for its constituents like vitamins $\mathrm{A}, \mathrm{B}, \mathrm{C}, \mathrm{E}$ and folic acid and minerals like calcium, sodium, potassium, magnesium, iron, copper and zinc. Studies demonstrated the antiviral, antibacterial and antifungal properties of Aloe vera and the use of this plant is favorable. ${ }^{43}$ This is effective against oral bacteria like Streptococcus mutans, Streptococcus mitis, Lactobacillus acidophilus, Prevotella intermedia and Enterococcus faecalis. In addition, the antiseptic ingredients like lupeol, urea nitrogen, salicylic acid, cinnamonic acid, phenol and sulphur has repressive action over fungi, bacteria and viruses. The extract of Aloe vera gel has inhibitory action on the arachidonic acid pathway via cyclooxygenase inhibiting inflammation, thus proving the anti-inflammatory action with analgesic properties. Studies have also shown the antioxidant effect of some of the constituents of Aloe vera gel. The isorabichromone, feruoyl aloesin, p-coumaroyl aloesin derivatives of aloesin from aloe revealed potent free radical and superoxide anion activity. Aloe vera gel has been tried in various case control studies for aphthous ulcer treatment and given excellent clinical results by its anti-inflammatory and immunomodulatory effects. ${ }^{22}$

\section{Myrrh (stem of different Commiphora species)}

Terpenoids, steroids, carbohydrates, flavonoids, lignans and long chain aliphatic derivatives are the significant phytochemicals found in various Myrrh species. The most important species of myrrh is Commiphora molmol which has been used as an effective antimicrobial agent. Myrrh has local stimulant, wound healing and antiseptic properties. The Myrrh extract is used for sore throats, canker sores and gingivitis, acne, boils and arthritis. It has been shown to possess anti-hyperglycemic effect in diabetic rat models. Myrrh reduces cholesterol and triglycerides and increased the SD activity in hypercholesterolemic rabbit models. It shows strong antithrombotic activity. ${ }^{44}$ Myrrh extract is used as digestive aid drug and it is used in food and oral healthcare drug products approved by FDA. In peritoneal macrophages, pretreatment with myrrh prevented formation of proinflammatory cytokines including IL- $1 \beta$, IL 6, and TNF- $\alpha$ as well as nitric oxide and PGE2. The analgesic effect of myrrh could be due to the furanoeudesma-1, 3-diene and terpene existing in myrrh troubles opioid receptors in the mouse's brain, manipulating pain perception. In dentistry, Myrrh has been utilized as anti-inflammatory, anti-infectious and wound-healing substance. Myrrh can reduce toothache when applied to teeth. ${ }^{22}$ 


\section{Lavender oil}

Mori et al, in their recent research on an animal model topically treated skin wounds with lavender oil, for alternate 14 days. Lavandula angustifolia is a well-known traditional medicine having antibacterial and antifungal properties and exhibited positive effects over burns and insect bites. It also has anxiolytic and anti-inflammatory properties. As matched to control, there is a rapid progress in the cutaneous wound closure with the topical application of lavender oil with increased expression of growth factors like PDGF-A and EGF playing essential role in tissue remodeling and re-epithelialization. The area of the wound was decreased significantly compared to control. In addition, it promoted the synthesis of collagen, differentiation of fibroblasts by upregulation of TGF- $\beta$. These are important for the early granulation tissue formation, tissue remodeling by collagen synthesis and late phase wound contraction. ${ }^{45}$

\section{Rosa damascene}

Rosa damascene has got analgesic, anti-inflammatory, antinociceptive and wound healing properties. The analgesic effect is due to the presence of non-water soluble ingredients such as Quercelin and Kaempferol. The phenolic compounds, Vitamin $\mathrm{C}$ and tannins possess antiinflammatory effects. The wound healing effect is mainly due to the initiation of growth factor release. $\beta$-citronellol, geraniol, nerol, nonadecane, and Kaempferol compounds could be accountable for the wound healing properties. ${ }^{46}$

\section{Myrtle (Myrtus communis)}

Mouth ulcers, fungal infection and cold have been treated with Myrtus communis leaves and fruits. The leaves are having anti-inflammatory, antioxidant, antibiotic or antiseptic effects. The main compounds of these plants are essential oils, phenolic compounds, tannins, flavonoids and proanthocyanidins. Various studies have explored the effectiveness of myrtle on aphthous ulcer as topical medicine in the forms of oral solution, paste and decoction. The clinical efficacy on aphthous ulcer is mainly due to its anti-inflammatory, analgesic, antiseptic, antiviral and antibacterial effects. The new ulcer formation can be prevented by the management of inflammatory cytokines, inhibiting the TNF- $\alpha$. It also has anti-nociceptive, anesthetic and wound healing properties which could progress the ulcer healing and decrease pain duration. The antimicrobial effects prevent secondary infections thereby improving the symptoms. ${ }^{47}$

\section{Camel thorn}

Camel thorn otherwise known as Alhagi Camelorum is a folk medicine used in Iran to treat gastric disorders. Camel thorn contains some flavanones, such as alhagitin and alhagidin. Studies have proved that the nitric oxide (NO) level is significantly higher in aphthous ulcer patients than that the individuals without RAU. Studies have stated that in oral aphthous ulcer patients, lipid peroxidation and NO can inhibit the wound healing activity of epidermal growth factor (EGF). It is recognized that flavanones could prevent macrophage and consequently the formation of NO and TNF- $\alpha$. This could result in improved and earlier healing of the aphthous ulcers. It has been implied that the plant has a potential to decrease the number of episodes of ulcers in patients with RAU. ${ }^{26}$

\section{Centella asiatica}

The biologically active component of Centella asiatica is triterpenes and saponis. The important compound of triterpenes includes Asiatic acid, medecassic acid, asiaticosside and madecassoside. It also contains flavonoids and volatile oils. It is popularly known for its antioxidative and neuroprotective effects. It was revealed that $0.50,1$ and $2 \%$ Centella asiatica gel and triamcinolone acetonide shown indifferent decreasing rate of lesion size with significantly greater rates in comparison with placebo $(p<0.05) .{ }^{48}$ A study report stated that enhanced wound healing ability of $C$. asiatica is by preventing inflammation, enhancing collagen production, supporting angiogenesis, promoting vasodilation and decreasing oxidative stress in the wound. The fibroblast growth factor and vascular endothelial growth factors are responsible for the wound healing activity. In injured tissues, the C. asiatica extracts has been revealed to disturb cellular growth and proliferation. ${ }^{27}$

\section{Citrus oil and magnesium salts}

The citrus oil is known to have anti-bacterial and anti-inflammatory properties. Magnesium has mild anesthetic properties. $\beta$-pinene and limonenes are the two major monoterpenes found in citrus lemon essential oil. This oil is well known for its anti ulcerogenic and antibacterial effects. The limonene might also have immunomodulatory effects. The magnesium salts has anti-nociceptive effects and it provides significant reduction in pain. A study report stated that the mucoadhesive patch containing citrus oil and magnesium salts, seal and defends the oral ulcer from oral pathogens and trauma. The patch is also effective in reduction in mean duration of pain and considerable improvement in oral functions after the treatment. ${ }^{49}$ The magnesium salt did not exhibit any antibacterial effects whereas citrus oil is known to have an antibacterial effect with the minimum inhibitory concentration (MIC) of $1 \mathrm{mg} / \mathrm{ml}$. The mixture effected in decreased levels of TNF- $\alpha$ and leukocyte migration while the levels of anti- inflammatory interleukin-10 is maintained. ${ }^{28}$

\section{Liquorice}

This is otherwise known as yashtimadhu, sweet wood, Atimaduram or mulhatti which belongs to the genus Glycyrrhiza which is known for its immense medicinal values. The bioactive ingredients of liquorice are glabridin, licoricidin, licorisoflavan A, licochalcone and glycyrrhizin. It is well identified for, anti-inflammatory, anti-ulcerative, antioxidant, antiviral; glucocorticoid, anti-carcinogenic and many more properties. ${ }^{50}$ A recent in vivo study assessed the clinical effectiveness of licorice bio adhesive hydrogel patches in recurrent aphthous ulcer patients. According to the study results, liquorice bio adhesive can be efficient in decreasing the pain, the inflammatory halo and the necrotic center of aphthous ulcers. The authors stated that the effect is mainly due to the anti-inflammatory effect of the liquorice. Among the 30 species of liquorice, the anti-inflammatory action is mainly concentrated on G. glabra and G. uralensis. The anti-inflammatory effects are increased superoxide dismutase enzymatic defense system of the colonic mucosa. There was a dose dependent reduction in TNF $\alpha$, NO and IL- 6 levels. It also decreases the MMPs, PGE2 and other free radicals. These effects are primarily due to the effects of triterpenoids and flavonoid compounds of liquorice. ${ }^{29}$

\section{CONCLUSION}

The isolation, characterization of active components, defining exact safety margin of drug, concentration of the active components, standardization and scientific validation are the most crucial factors to be considered while designing the herbal medicine. Herbal medications are the most preferred and well accepted drugs by the patients in a few clinical scenarios. In general, the topical herbal medications were effective over minor aphthous ulcer in various clinical outcomes within the study limits. The recent clinical studies have focused on herbal mucoadhesive patches for topical interventions of aphthous ulcer. All the topical herbal preparations were clinically effective in reducing the ulcer symptoms with improved healing. Most herbs share few common biological actions like anti-inflammatory, analgesic and immunomodulatory effects. There 
is scarcity of data to warrant the clinical efficacy of combined systemic administration and topical application of the same herbal drugs in repeated cases. Further evidence based research is needed for the effective use of the herbal therapy in repeated cases of RAS. Research over the ability of topical herbal application to manage other kinds of acute painful oral ulcers can be recommended.

\section{REFERENCES}

1. Hamedi S, Sadeghpour O, Shamsardekani MR, Amin G, Hajighasemali D, Feyzabadi Z. The most common herbs to cure the most common ora disease: Stomatitis recurrent aphthous ulcer (RAU). Iran Red Crescent Med J. 2016;18(2):e21694. doi: 10.5812/ircmj.21694, PMID 27186385.

2. Hargitai IA. Painful oral lesions. Dent Clin North Am. 2018;62(4):597-609. doi: 10.1016/j.cden.2018.06.002, PMID 30189985.

3. Sabbagh $A H$, Felemban MF. Therapeutic management of recurrent aphthous stomatitis: A review of the growing knowledge. Ann Int Med Dent Res. 2016;2(6):1-9. doi: 10.21276/aimdr.2016.2.6.DE1.

4. Pak Fetrat A, Haddadi M, Setayesh Y, Shirazi AS, Moeintaghavi A. Natura Treatment of Oral Aphthous Ulcers: A Systematic Review. Transl Biomed;08(4). doi: 10.21767/2172-0479.100138

5. Edgar NR, Saleh D, Miller RA. Recurrent aphthous stomatitis: A review. J Clin Aesthet Dermatol. 2017;10(3):26-36. PMID 28360966.

6. Challacombe SJ, Alsahaf S, Tappuni A. Recurrent Aphthous Stomatitis: Towards evidence-based Treatment? Curr Oral Health Rep. 2015;2(3):158-67. doi: $10.1007 / s 40496-015-0054-y$.

7. Li C, Huang H, Hua H, Wang W. Efficacy and safety of topical herbal medicine treatment on recurrent aphthous stomatitis: a\&nbsp;systemic review. Drug Des Dev Ther. doi: 10.2147/DDDT.S96589.

8. Sharquie KE, Noaimi AA, Latif TM. Treatment of recurrent aphthous stomatitis by $100 \%$ topical pumpkin seed oil. J Cosmet Dermatol Sci Appl. 2017;07(4):324-35. doi: 10.4236/jcdsa.2017.74029

9. Khuje PD, Hulke SM. Consumption of Yogurt, Aloe-vera pulp and Holy Basil leaves to reduce the recurrence of aphthous mouth ulcers in night shift workers. Int J Appl Res. 2017;3(4):807-9.

10. Vaziri S, Mojarrab M, Farzaei MH, Najafi F, Ghobadi A. Evaluation of antiaphthous activity of decoction of Nicotina tobacum leaves as a mouthwash: A placebo-controlled clinical study. J Tradit Chin Med. 2016;15(36 (2)):160-4.

11. Haghpanah P, Moghadamnia AA, Zarghami A, Motallebnejad M. Mucobioadhesive containing Ginger officinale extract in the management of recurrent aphthous stomatitis: A randomized clinical study. Caspian J Intern Med. 2015;6(1):3-8. PMID 26221489.

12. Romero-Cerecero O, Zamilpa A, Tortoriello J. Pilot study that evaluated the clinical effectiveness and safety of a phytopharmaceutical elaborated with an extract of Ageratina pichinchensis in patients with minor recurrent aphthous stomatitis. J Ethnopharmacol. 2015;173:225-30. doi: 10.1016/j.jep.2015.06.021, PMID 26102552

13. Nair PK, Dyasanoor S. Clinical efficacy of allicin - A novel alternative therapeutic agent in the management of minor recurrent aphthous stomatitis. JCRI. 2015;2(6):231-6. doi: 10.15713/ins.jcri.85.

14. Gavanji S, Larki B, Bakhtari A. The effect of extract of Punica granatum var pleniflora for treatment of minor recurrent aphthous stomatitis. Integr Med Res. 2014;3(2):83-90. doi: 10.1016/j.imr.2014.03.001, PMID 28664082.

15. Seyyedi SA, Sanatkhani M, Pakfetrat A, Olyaee P. The therapeutic effects of Chamomilla tincture mouthwash on oral aphthae: A randomized clinical trial. J Clin Exp Dent. 2014;6(5):e535-8. doi: 10.4317/jced.51472, PMID 25674322

16. Khademi H, Iranmanesh P, Moeini A, Tavangar A. Evaluation of the effectiveness of the Iralvex gel on the recurrent aphthous stomatitis management. Int Sch Res Notices. 2014;2014:175378. doi: 10.1155/2014/175378.

17. HeY, gong D, Zhu R. The efficacy of Kasmitad gel in the management of recurrent minor aphthous ulceration. Kuwait Med J. 2014;46(2):124-9.

18. Stojanovska AA, Popovska M, Muratvska I, Mitic K, Stefanovska E, Nikolovska VR. Therapeutic effect of Proaftal in the treatment of recurrent aphthous stomatitis. Prilozi. 2015;35(3):195-202.

19. Deshmukh RA, Bagewadi AS. Comparison of effectiveness of curcumin with triamcinolone acetonide in the gel form in treatment of minor recurrent aphthous stomatitis: A randomized clinical trial. Int J Pharm Investig. 2014;4(3):138-41. doi: 10.4103/2230-973X.138346, PMID 25126527.

20. Guintu FZ, Chua AH. Effectivity of guava leaves (Psidium guajava) as mouthwash for patients with aphthous ulcers. Philipp J Otolaryngol Head Neck Surg. 2013;28(2):8-13. doi: 10.32412/pjohns.v28i2.469.

21. Jiang $X W$, Zhang $Y$, Zhu YL, Zhang $H$, Lu $K$, Li FF, et al. Effects of berberine gelatin on recurrent aphthous stomatitis: A randomized, placebo-controlled, double-blind trial in a Chinese cohort. Oral Surg Oral Med Oral Pathol Ora Radiol. 2013;115(2):212-7. doi: 10.1016/j.000o.2012.09.009, PMID 23246229.
22. Mansour G, Ouda S, Shaker A, Abdallah HM. Clinical efficacy of new Aloe vera- and myrrh-based oral mucoadhesive gels in the management of minor recurrent aphthous stomatitis: A randomized, double-blind, vehicle-controlled study, J Oral Pathol Med. 2014:43(6):405-9. doi: 10.1111/jop.12130, PMID 24164309.

23. Altaei DT. Topical lavender oil for the treatment of recurrent aphthous ulceration Am J Dent. 2012;25(1):39-43. PMID 22558691.

24. Hoseinpour H, Peel SA, Rakhshandeh H, Forouzanfar A, Taheri M, Rajabi O, et al. Evaluation of Rosa damascena mouthwash in the treatment of recurrent aphthous stomatitis: A randomized, double-blinded, placebo-controlled clinical trial. Quintessence Int. 2011;42(6):483-91. PMID 21519586.

25. Babaee N, Mansourian A, Momen-Heravi F, Moghadamnia A, Momen-Beitollahi J. The efficacy of a paste containing Myrtus communis (Myrtle) in the management of recurrent aphthous stomatitis: A randomized controlled trial. Clin Ora Investig. 2010;14(1):65-70. doi: 10.1007/s00784-009-0267-3, PMID 19306024.

26. Pourahmad $M$, Rahiminejad $M$, Fadaei $S$, Kashafi $H$. Effects of camel thorn distillate on recurrent oral aphthous lesions. J Dtsch Dermatol Ges. 2010;8(5):348-52. doi: 10.1111/j.1610-0387.2010.07316.x, PMID 20136677.

27. Ruengprasertkit $\mathrm{C}$, Hongprasong $\mathrm{N}$, Tantisirs $\mathrm{MH}$. Preliminary study of effects of a standardized extract of Centella asiatica ECa 233 on minor aphthous ulcers. CU Dent J. 2010;33:131-42.

28. Shemer A, Amichai B, Trau H, Nathansohn N, Mizrahi B, Domb AJ. Efficacy of a mucoadhesive patch compared with an oral solution for treatment of aphthous stomatitis. Drugs R D. 2008;9(1):29-35. doi: 10.2165/00126839-20080901000003, PMID 18095751.

29. Moghadamnia AA, Motallebnejad M, Khanian M. The efficacy of the bioadhesive patches containing licorice extract in the management of recurrent aphthous stomatitis. Phytother Res. 2009;23(2):246-50. doi: 10.1002/ptr.2601, PMID 18853400.

30. Bardaa S, Ben Halima N, Aloui F, Ben Mansour R, Jabeur H, Bouaziz M, et al. Oil from pumpkin (Cucurbita pepo L.) seeds: Evaluation of its functional properties on wound healing in rats. Lipids Health Dis. 2016;15(1):73. doi: 10.1186/s12944016-0237-0

31. Mohan L, Amberkar MV, Kumari M. Ocimum sanctum Linn (Tulsi)-an overview. Int PharmSci Rev Res. 2011;7(1):51-3.

32. Vaseem A, Subhani G, Afshan K, Mohammed MA, Khan MA, Rumana MT. Effect of Ocimum sanctum linn in stress induced gastric ulcers in rats. Int J Med Res Health Sci. 2015;4(3):616-21.

33. Kadian R, Parle M. Therapeutic potential and phytopharmacology of tulsi. Int J Pharm Life Sci. 2012;3(7):1858-67.

34. Rawat A, Mali RR. Phytochemical and pharmacological activities of Nicotina tobacum: A review. Indian J Pharm Biol Res. 2013;1(2):74-82.

35. Gupta R, Singh PK, Singh R, Singh RL. Pharmacological activities of Zingiber officinale (Ginger) and its active ingredients: A review. Int J Scilnnov. Res.2016;4:1-18.

36. Du Q, Ni S, Guo L, Song W, Zhao K, Liu N, et al. Effects of Zingiber officinalis (WILLD) ROSC. Mebranes on minor recurrent aphthous stomatitis: A randomized pragmatic trial. J Tradit Chin Med Sci. 2018;5(1):58-63.

37. Novianty RA, Chrismawaty BE, Subagyo G. Effect of allicin for re-epithelialization during healing in oral ulcer model. The Indonesian J Dent Res. 2011:1(2):87-93. doi: 10.22146/theindjdentres.9990.

38. Xu X, Yin $\mathrm{P}$, Wan $\mathrm{C}$, Chong $\mathrm{X}$, Liu $\mathrm{M}$, Cheng $\mathrm{P}$, et al. Punicalagin inhibits inflammation in LPS-induced RAW264.7 macrophages via the suppression of TLR4-mediated MAPKs and NF-אB activation. Inflammation. 2014;37(3):956-65. doi: 10.1007/s10753-014-9816-2, PMID 24473904.

39. Ramos-e-Silva M, Ferreira AF, Bibas R, Carneiro S. Clinical evaluation of fluid extract of Chamomilla recutita for oral aphthae. J Drugs Dermato 2006:5(7):612-7. PMID 16865865.

40. Wagh VD. Propolis: A wonder bee's product and its pharmacological potentials. Adv Pharmacol Sci. 2013;2013:308249. doi: 10.1155/2013/308249.

41. Akbik D, Ghadiri M, Chrzanowski W, Rohanizadeh R. Curcumin as a wound healing agent. Life Sci. 2014;116(1):1-7. doi: 10.1016/j.Ifs.2014.08.016, PMID 25200875.

42. Naseer S, Hussain S, Naeem N, Pervaiz M, Rahman M. The phytochemistry and medicinal value of Psidium guajava (guava). Clin Phytosci. 2018:4(1):32. doi: 10.1186/s40816-018-0093-8

43. Radha $\mathrm{MH}$, Laxmipriya NP. Evaluation of biological properties and clinica effectiveness of Aloe vera: A systematic review. J Tradit Complement Med. 2015;5(1):21-6. doi: 10.1016/j.jtcme.2014.10.006, PMID 26151005.

44. Shen T, Li GH, Wang XN, Lou HX. The genus Commiphora: A review of its traditional uses, phytochemistry and pharmacology. J Ethnopharmacol. 2012;142(2):319-30. doi: 10.1016/j.jep.2012.05.025, PMID 22626923.

45. Mori HM, Kawanami $H$, Kawahata H, Aoki M. Wound healing potential of lavender oil by acceleration of granulation and wound contraction through induction of TGF- $\beta$ in a rat model. BMC Complement Altern Med. 2016;16:144. doi: 10.1186/s12906-016-1128-7, PMID 27229681.

46. Mahabob N, Mohan J, Gunasekaran S. Clinical effectiveness of Rosa damascena mill in the management of oral mucosal lesions. World J Pharm Res. 2018;4(9):155-60. 
47. Mahboubi M. Myrtus communis L. and its application in treatment of Recurrent Aphthous Stomatitis. J Ethnopharmacol. 2016;193:481-9. doi: 10.1016/j. jep.2016.09.054, PMID 27693775.

48. Krassanai W. Treatment of recurrent oral aphthous ulcerations. Ann Dent UM.1999;6(1):58-9.

49. Kürklü-Gürleyen E, Ögüt-Erisen M, Çakır O, Uysal Ö, Ak G. Quality of life in patients with recurrent aphthous stomatitis treated with a mucoadhesive patch containing citrus essential oil. Patient Prefer Adherence. 2016;10:967-73. doi: 10.2147/PPA.S106530, PMID 27313450.

50. Sidhu P, Shankargouda S, Rath A, Hesarghatta Ramamurthy P, Fernandes B, Kumar Singh A. Therapeutic benefits of liquorice in dentistry. J Ayurveda Integr Med. 2020;11(1):82-8. doi: 10.1016/j.jaim.2017.12.004, PMID 30391123.

Article History: Received: 10-05-2021; Revised: 17-09-2021; Accepted: 25-10-2021.

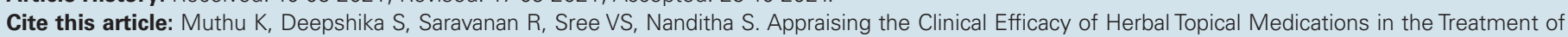
Recurrent Aphthous Stomatitis - A Review. J Young Pharm. 2021;13(4):342-51. 